\title{
New Properties of Dual Continuous K-g-Frames in Hilbert Spaces
}

\author{
Abdeslam Touri $\mathbb{i}$, ${ }^{1}$ Hatim Labrigui $\mathbb{D},{ }^{1}$ and Mohamed Rossafi $\mathbb{D}^{2}$ \\ ${ }^{1}$ Department of Mathematics, University of Ibn Tofail, B. P. 133, Kenitra, Morocco \\ ${ }^{2}$ LASMA Laboratory Department of Mathematics, Faculty of Sciences Dhar El Mahraz, University Sidi Mohamed Ben Abdellah, \\ Fes, Morocco \\ Correspondence should be addressed to Abdeslam Touri; touri.abdo68@gmail.com
}

Received 30 December 2020; Revised 10 March 2021; Accepted 28 March 2021; Published 12 April 2021

Academic Editor: Seppo Hassi

Copyright (c) 2021 Abdeslam Touri et al. This is an open access article distributed under the Creative Commons Attribution License, which permits unrestricted use, distribution, and reproduction in any medium, provided the original work is properly cited.

The concept of frames in Hilbert spaces continues to play a very interesting role in many kinds of applications. In this paper, we study the notion of dual continuous $K$-g-frames in Hilbert spaces. Also, we establish some new properties.

\section{Introduction and Preliminaries}

The concept of frames in Hilbert spaces was introduced by Duffin and Schaeffer in 1952 [1] to study some deep problems in nonharmonic Fourier series; after the fundamental paper [2] by Daubechies, Grossman, and Meyer, frames' theory began to be widely used, particularly in the more specialized context of wavelet frames and Gabor frames [3].

Let $X$ be a Banach space, $(\Omega, \mu)$ a measure space, and function $f: \Omega \longrightarrow X$ a measurable function. The integral of the Banach-valued function $f$ was defined by Bochner and others. Most properties of this integral are similar to those of the integral of real-valued functions.

Let $(\Omega, \mu)$ be a measure space, let $U$ and $V$ be two separable Hilbert spaces, $\left\{V_{w}\right\}_{w \in \Omega}$ is a sequence of subspaces of $V$, we denote $\mathscr{B}(U, V)$ as the collection of all bounded linear operators from $U$ to $V$, and $\mathscr{B}(U, U)$ is abbreviated to $\mathscr{B}(U)$.

For $T \in \mathscr{B}(U, V)$, we use the notation $\mathscr{R}(T)$ and $\mathcal{N}(T)$ to denote, respectively, the range and the null space of $T$.

Definition 1 (see [4]). A sequence $\left\{\Lambda_{\omega} \in \mathscr{B}\left(U, V_{\omega}\right)\right\}_{\omega \in \Omega}$ is called a continuous g-frame for $U$ with respect to $\left\{V_{\omega}\right\}_{\omega \in \Omega}$ if there exists $0<A<B<\infty$ such that

$$
A\|x\|^{2} \leq \int_{\Omega}\left\|\Lambda_{\omega} x\right\|^{2} \mathrm{~d} \mu(\omega) \leq B\|x\|^{2}, \quad \forall x \in U .
$$

The numbers $A$ and $B$ are called lower and upper bounds of the continuous g-frame, respectively.

If $A=B=\lambda$, the frame is $\lambda$-tight.

If $A=B=1$, it is called a normalized tight continuous g-frame or a Parseval continuous g-frame.

We call $\left\{\Lambda_{\omega} \in \mathscr{B}\left(U, V_{\omega}\right)\right\}_{\omega \in \Omega}$ a continuous g-Bessel sequence if the right-hand inequality of (1) holds.

Let $\Lambda=\left\{\Lambda_{w}\right\}_{\in \Omega}$ be a continuous g-frame for $U$ with respect to $\left\{V_{\omega}\right\}_{\omega \in \Omega}$.

The synthesis operator of $\Lambda$ is defined by $T_{\Lambda}: \oplus_{\omega \in \Omega} V_{w} \longrightarrow U$, given by

$$
T_{\Lambda}\left(\left\{y_{w}\right\}_{w \in \Omega}\right)=\int_{\Omega} \Lambda_{\omega}^{*} y_{\omega} \mathrm{d} \mu(w), \quad \forall\left\{y_{w}\right\}_{w \in \Omega} \in \underset{\omega \in \Omega}{\oplus} V_{w},
$$

where $\oplus_{\omega \in \Omega} V_{w}=\left\{\left(y_{w}\right)_{w \in \Omega}, y_{\omega} \in V_{\omega} ;\left\|\int_{\Omega}\left|y_{\omega}\right|^{2} \mathrm{~d} \mu(\omega)\right\|<\right.$ $\infty\}$.

The existence of $\int_{\Omega} \Lambda_{\omega}^{*} y_{\omega} \mathrm{d} \mu(w)$ implies that $T_{\Lambda}$ is well defined and bounded.

The adjoint operator of $T_{\Lambda}$ which is given by

$$
T_{\Lambda}^{*}: U \longrightarrow \underset{\omega \in \Omega}{\oplus} V_{w} ; T_{\Lambda}^{*}(x)=\left\{\Lambda_{\omega} x\right\}_{w \in \Omega}, \quad \forall x \in U
$$

is said to be the analysis operator of $\left\{\Lambda_{w}\right\}_{\omega \in \Omega}$. 
Definition 2 (see [5]). Let $K \in \mathscr{B}(U)$. A sequence $\left\{\Lambda_{\omega} \in \mathscr{B}\left(U, V_{\omega}\right)\right\}_{\omega \in \Omega}$ is called a continuous $K$-g-frame for $U$ with respect to $\left\{V_{\omega}\right\}_{\omega \in \Omega}$ if there exists $0<A<B<\infty$ such that

$$
A\left\|K^{*} x\right\|^{2} \leq \int_{\Omega}\left\|\Lambda_{\omega} x\right\|^{2} \mathrm{~d} \mu(\omega) \leq B\|x\|^{2}, \quad \forall x \in U .
$$

The numbers $A$ and $B$ are called lower and upper bounds of the continuous $K$-g-frame, respectively.

If $A\left\|K^{*} x\right\|^{2}=\int_{\Omega}\left\|\Lambda_{\omega} x\right\|^{2} \mathrm{~d} \mu(\omega)$, the sequence $\left\{\Lambda_{\omega} x\right\}_{w \in \Omega}$ is called a continuous $K$-g-frame for $U$ with respect to $\left\{V_{\omega}\right\}_{\omega \in \Omega}$.

The $A$-tight continuous $K$-g-frame for $U$ is said to be a Parseval continuous $K$-g-frame if $A=1$.

Suppose that $K \in \mathscr{B}(U)$ and $\Lambda=\left\{\Lambda_{\omega} \in \mathscr{B}\left(U, V_{\omega}\right)\right\}_{\omega \in \Omega}$ is a Parseval continuous $K$-g-frame for $U$ with respect to $\left\{V_{\omega}\right\}_{\omega \in \Omega}$ with synthesis operator $T_{\Lambda}$. Then, it is easy to check

$$
K K^{*} x=T_{\Lambda} T_{\Lambda} x=\int_{\Omega} \Lambda_{\omega}^{*} \Lambda_{\omega} x \mathrm{~d} \mu(\omega), \quad \forall x \in U
$$

Definition 3. Let $K \in \mathscr{B}(U)$ and $\left\{\Lambda_{\omega}\right\}_{\omega \in \Omega} \in \mathscr{B}\left(U, V_{\omega}\right)$ be a continuous $K$-g-frame for $U$ with respect to $\left\{V_{\omega}\right\}_{\omega \in \Omega}$. A continuous $K$-g-Bessel sequence $\left\{\Gamma_{\omega}\right\}_{\omega \in \Omega}$ for $U$ with respect to $\left\{V_{\omega}\right\}_{\omega \in \Omega}$ is said to be a dual continuous $K$-g-Bessel sequence of $\left\{\Lambda_{\omega}\right\}_{\omega \in \Omega}$ if

$$
K x=\int_{\Omega} \Lambda_{\omega}^{*} \Gamma_{\omega} x \mathrm{~d} \mu(w), \quad \forall x \in U .
$$

\section{Remark 1}

(1) If $K=I d_{U}$, then a dual continuous $K$-g-Bessel sequence is just a dual continuous g-frame

(2) A dual continuous $K$-g-Bessel sequence is necessarily a continuous $K^{*}$-g-frame

(3) Denote the synthesis operator of $\left\{\Lambda_{\omega}\right\}_{\omega \in \Omega}$ and $\left\{\Gamma_{\omega}\right\}_{\omega \in \Omega}$ by $T_{\Lambda}$ and $T_{\Gamma}$, respectively; then, (6) means that $K=T_{\Lambda} T_{\Gamma}^{*}$

Lemma 1 (see [6]). Suppose that $T \in \mathscr{B}(U, V)$ has a closed range; then, there exists a unique operator $T^{+} \in \mathscr{B}(U, V)$, called the pseudo-inverse of $T$, satisfying

$$
\begin{aligned}
T T^{+} T & =T, \\
T^{+} T T^{+} & =T^{+}, \\
\left(T T^{+}\right)^{*} & =T T^{+}, \\
\left(T^{+} T\right)^{*} & =T^{+} T, \\
\left(T^{*}\right)^{+} & =\left(T^{+}\right)^{*} \mathcal{N}\left(T^{+}\right)=(\mathscr{R}(T))^{\perp}, \\
\mathscr{R}\left(T^{+}\right) & =(\mathcal{N}(T))^{\perp} .
\end{aligned}
$$

Lemma 2 (see [7]). Let $U, U_{1}$, and $U_{2}$ be three Hilbert spaces; also, let $T \in \mathscr{B}\left(U_{1}, U\right)$ and $K \in \mathscr{B}\left(U_{2}, U\right)$. The following statements are equivalent:
(1) $\mathscr{R}(T) \subset \mathscr{R}(K)$

(2) There exists $\lambda>0$ such that $T T^{*} \leq \lambda K K^{*}$

(3) There exists $\theta \in \mathscr{B}\left(U_{1}, U_{2}\right)$ such that $T=K \theta$

Moreover, if (1)-(3) are valid, then there exists a unique operator $\theta$ such that

(a) $\|\theta\|^{2}=\inf \left\{\mu: T T^{*} \leq \mu K K^{*}\right\}$

(b) $\mathscr{N}(T)=\mathscr{N}(\theta)$

(c) $\mathscr{R}(\theta) \subset \overline{\mathscr{R}\left(K^{*}\right)}$

In the following section, we set out to generalize some results of Xiang [8]; in other words, we characterize the concept of dual continuous $K$-g-frames in Hilbert spaces, and we give some properties. Our results extend, generalize, and improve many existing results.

\section{Main Result}

Proposition 1. Suppose that $K \in \mathscr{B}(U)$ has a closed range, and let $\Lambda=\left\{\Lambda_{\omega} \in \mathscr{B}\left(U, V_{\omega}\right)\right\}_{\omega \in \Omega}$ to be a continuous $K-g$ frame for $U$ with respect to $\left\{V_{\omega}\right\}_{\omega \in \Omega}$ and $\Gamma=\left\{\Gamma_{\omega}\right\}_{\omega \in \Omega}$ be a dual continuous $K$ - $g$-Bessel sequence of $\left\{\Lambda_{\omega}\right\}_{\omega \in \Omega}$; then, $\left\{\Gamma_{\omega} K^{*}\right\}_{\omega \in \Omega}$ is a continuous $K$-g-frame for $U$ with respect to $\left\{V_{\omega}\right\}_{\omega \in \Omega}$.

Proof. For each $x \in U$, we have $K x=\int_{\Omega} \Lambda_{\omega}^{*} \Gamma_{\omega} x \mathrm{~d} \mu(w)$, and then

$$
\begin{aligned}
\|K x\|^{2} & =\sup _{\|y\|=1}|\langle K x, y\rangle|^{2} \\
& =\sup _{\|y\|=1}\left|\int_{\Omega}\left\langle\Gamma_{\omega} x, \Lambda_{\omega} y\right\rangle \mathrm{d} \mu(\omega)\right|^{2} \\
& \leq \sup _{\|y\|=1} \int_{\Omega}\left\|\Gamma_{\omega} x\right\|^{2} \mathrm{~d} \mu(\omega) \int_{\Omega}\left\|\Lambda_{\omega} y\right\|^{2} \mathrm{~d} \mu(\omega) \\
& \leq B_{\Lambda} \int_{\Omega}\left\|\Gamma_{\omega} x\right\|^{2} \mathrm{~d} \mu(\omega),
\end{aligned}
$$

which give

$$
B_{\Lambda}^{-1}\|K x\|^{2} \leq \int_{\Omega}\left\|\Gamma_{\omega} x\right\|^{2} \mathrm{~d} \mu(\omega) \leq B_{\Gamma}\|x\|^{2},
$$

where $B_{\Lambda}$ and $B_{\Gamma}$ are the upper bounds of $\Lambda$ and $\Gamma$, respectively.

Consequently,

$$
B_{\Lambda}^{-1}\left\|K K^{*} x\right\|^{2} \leq \int_{\Omega}\left\|\Gamma_{\omega} K^{*} x\right\|^{2} \mathrm{~d} \mu(\omega) \leq B_{\Gamma}\left\|K^{*} x\right\|^{2} \leq B_{\Gamma}\|K\|^{2}\|x\|^{2} .
$$

Assume that $K^{*}$ has a closed range; hence, for each $y \in \mathscr{R}\left(K^{*}\right)$, we get $y=K^{*}\left(K^{*}\right)^{+} y=K^{+} K y$, by Lemma 1 , and thus,

$$
\|y\|^{2}=\left\|K^{+} K y\right\|^{2} \leq\left\|K^{+}\right\|^{2}\|K y\|^{2} .
$$

Now, 


$$
B_{\Lambda}^{-1}\left\|K^{+}\right\|^{-2}\left\|K^{*} x\right\|^{2} \leq B_{\Lambda}^{-1}\left\|K K^{*} x\right\|^{2} \leq \int_{\Omega}\left\|\Gamma_{\omega} K^{*} x\right\|^{2} \mathrm{~d} \mu(\omega) \leq B_{\Gamma}\|K\|^{2}\|x\|^{2},
$$

which gives $\left\{\Gamma_{\omega} K^{*}\right\}_{\omega \in \Omega}$ is a continuous $K$-g-frame for $U$ with respect to $\left\{V_{\omega}\right\}_{\omega \in \Omega}$ with bounds $B_{\Lambda}^{-1}\left\|K^{+}\right\|^{-2}$ and $B_{\Gamma}\|K\|^{2}$.

Proposition 2. Let $K \in \mathscr{B}(U)$; then, every continuous $K-g$ frame admits a dual continuous $K-g$-Bessel sequence.
Proof. Let $\Lambda=\left\{\Lambda_{\omega} \in \mathscr{B}\left(U, V_{\omega}\right)\right\}_{\omega \in \Omega}$ be a continuous $K$-gframe for $U$ with respect to $\left\{V_{\omega}\right\}_{\omega \in \Omega}$, with bounds $C_{\Lambda}$ and $D_{\Lambda}$; then, for each $x \in U$, we have

$$
C_{\Lambda}\left\langle K^{*} x, K^{*} x\right\rangle=C_{\Lambda}\left\|K^{*} x\right\|^{2} \leq \int_{\Omega}\left\|\Lambda_{\omega} x\right\|^{2} \mathrm{~d} \mu(\omega)=\left\|T_{\Lambda}^{*} x\right\|^{2}=\left\langle T_{\Lambda}^{*} x, T_{\Lambda}^{*} x\right\rangle,
$$

which is equivalent to $C_{\Lambda} K K^{*} \leq T_{\Lambda} T_{\Lambda}^{*}$; by Lemma 2 , there exists $\theta \in \mathscr{B}\left(U, \oplus_{\omega \in \Omega} V_{w}\right)$ such that $K=T_{\Lambda} \theta$.

Let $P_{\omega}$ be the projection on $\oplus_{\omega \in \Omega} V_{w}$ that maps each element to its $w$-th component, i.e., $P_{\omega}\left(\left\{y_{z}\right\}_{z \in \Omega}\right)=\left\{u_{z}\right\}_{z \in \Omega}$, where $u_{z}=y_{\omega}$ if $z=\omega$, and $u_{z}=0$ if not.

If we let $\Gamma_{\omega}=P_{\omega} \theta$, then

$$
\begin{aligned}
\int_{\Omega}\left\|\Gamma_{\omega} x\right\|^{2} \mathrm{~d} \mu(\omega) & =\int_{\Omega}\left\|P_{\omega} \theta x\right\|^{2} \mathrm{~d} \mu(\omega) \\
& =\int_{\Omega}\left\|(\theta x)_{\omega}\right\|^{2} \mathrm{~d} \mu(\omega)=\|\theta x\|^{2} \\
& \leq\|\theta\|^{2}\|x\|^{2}, \quad \forall x \in U .
\end{aligned}
$$

Hence, $\left\{\Gamma_{\omega}\right\}_{\omega \in \Omega}$ is a continuous g-Bessel sequence for $U$ with respect to $\left\{V_{\omega}\right\}_{\omega \in \Omega}$. Now,

$$
K x=T_{\Lambda} \theta x=\int_{\Omega} \Lambda_{\omega}^{*}(\theta x)_{\omega} \mathrm{d} \mu(\omega)=\int_{\Omega} \Lambda_{\omega}^{*}\left(P_{\omega} \theta\right) x \mathrm{~d} \mu(\omega), \quad \forall x \in U,
$$

showing that $\left\{\Gamma_{\omega}\right\}_{\omega \in \Omega}$ is a continuous g-Bessel sequence.

Proposition 3. Suppose that $K \in \mathscr{B}(U)$ and $\Lambda=\left\{\Lambda_{\omega} \in \mathscr{B}\left(U, V_{\omega}\right)\right\}_{\omega \in \Omega}$ is a continuous $K$-g-frame for $U$ with respect to $\left\{V_{\omega}\right\}_{\omega \in \Omega}$; then, there exists a unique dual continuous $K$-Bessel sequence $\Gamma=\left\{\Gamma_{\omega}\right\}_{\omega \in \Omega}$ of $\Lambda$ such that $\left\|T_{\Gamma}\right\| \leq\left\|T_{\theta}\right\|$ for any dual continuous $K$-g-Bessel sequence $\Theta=$ $\left\{\theta_{\omega}\right\}_{\omega \in \Omega}$ of $\Lambda$.

Proof. Since $K K^{*} \leq\left(1 / C_{\Lambda}\right) T_{\Lambda} T_{\Lambda}^{*}$, by Lemma 2, it follows that there is a unique operator $R \in \mathscr{B}\left(U, \oplus_{\omega \in \Omega} V_{w}\right)$ such that $K=T_{\Lambda} R$ and

$$
\|R\|^{2}=\inf \left\{\lambda:\left\|K^{*} x\right\|^{2} \leq \lambda\left\|T_{\Lambda}^{*} x\right\|^{2}\right\},
$$

taking $\Gamma_{\omega}=P_{\omega} R$ for each $\omega \in \Omega$; then, as shown in Proposition $2, \Gamma$ is a continuous g-Bessel sequence for $U$ with respect to $\left\{V_{\omega}\right\}_{\omega \in \Omega}$, and furthermore, it is a dual continuous $K$-g-Bessel sequence of $\Lambda$. $T_{\Gamma}^{*}=R$.
Now, let $\left\{\theta_{\omega}\right\}_{\omega \in \Omega}$ be any dual continuous $K$-g-Bessel sequence of $\left\{\Lambda_{\omega}\right\}_{\omega \in \Omega}$; then, $K=T_{\Lambda} T_{\theta}^{*}$, and $K^{*}=T_{\theta} T_{\Lambda}^{*}$ as a sequence. For each $x \in U$, we have

$$
\left\|K^{*} x\right\|^{2}=\left\|T_{\theta} T_{\Lambda}^{*} x\right\|^{2} \leq\left\|T_{\theta}\right\|^{2}\left\|T_{\Lambda}^{*} x\right\|^{2} .
$$

It follows that $\left\|T_{\theta}\right\|^{2} \geq\|R\|^{2}=\left\|T_{\Gamma}^{*}\right\|^{2}=\left\|T_{\Gamma}\right\|^{2}$.

Equivalently, $\left\|T_{\theta}\right\| \geq\left\|T_{\Gamma}\right\|$.

Proposition 4. Suppose that $K \in \mathscr{B}(U)$ and $\Lambda=\left\{\Lambda_{\omega}\right\}_{\omega \in \Omega}$ is a continuous $K-g$-frame for $U$ with respect to $\left\{V_{\omega}\right\}_{\omega \in \Omega}$; then, the dual continuous $K-g$-Bessel sequence of $\Lambda$ is precisely the family $\left\{\Gamma_{\omega}\right\}_{\omega \in \Omega}=\left\{P_{\omega} \theta^{*}\right\}_{\omega \in \Omega}$, where $\theta \in \mathscr{B}\left(\oplus_{\omega \in \Omega} V_{w}, U\right)$ satisfies the condition $K=T_{\Lambda} \theta^{*}$.

Theorem 1. Let $K \in \mathscr{B}(U)$ and $\Lambda=\left\{\Lambda_{\omega}\right\}_{\omega \in \Omega}$ be a Parseval continuous $K$-g-frame for $U$ with respect to $\left\{V_{\omega}\right\}_{\omega \in \Omega}$, where $K$ has a closed range; then, $\left\{\Lambda_{\omega}\left(K^{+}\right)^{*}\right\}_{\omega \in \Omega}$ is a dual continuous $K$ - $g$-Bessel sequence of $\Lambda=\left\{\Lambda_{\omega}\right\}_{\omega \in \Omega}$.

Proof. It is easy to see that $\left\{\Lambda_{\omega}\left(K^{+}\right)^{*}\right\}_{\omega \in \Omega}$ is a continuous $K$ g-frame for $U$ with respect to $\left\{V_{\omega}\right\}_{\omega \in \Omega}$.

By Lemma 1, $y=K^{*}\left(K^{*}\right)^{+} y=K^{*}\left(K^{+}\right)^{*} y$ for every $y \in \mathscr{R}\left(K^{*}\right)$; thus, by (5),

$$
K y=K K^{*}\left(K^{+}\right)^{*} y=\int_{\Omega} \Lambda_{\omega}^{*} \Lambda_{\omega}\left(K^{+}\right)^{*} y \mathrm{~d} \mu(\omega) .
$$

If $h \in\left(\mathscr{R}\left(K^{*}\right)\right)^{\perp}=\mathscr{N}(K)$, then by Lemma 1 again, we obtain $h \in \mathscr{N}\left(\left(K^{*}\right)^{+}\right)$, implying that $\int_{\Omega} \Lambda_{\omega}^{*} \Lambda_{\omega}\left(K^{+}\right)^{*} h \mathrm{~d} \mu(\omega)=0=K h$. Altogether, we have

$$
K x=\int_{\Omega} \Lambda_{\omega}^{*} \Lambda_{\omega}\left(K^{+}\right)^{*} x \mathrm{~d} \mu(\omega), \quad \forall x \in U,
$$

which ends the proof.

Theorem 2. Let $K \in \mathscr{B}(U)$ have a closed range and $\Lambda=$ $\left\{\Lambda_{\omega}\right\}_{\omega \in \Omega}$ be a Parseval continuous K-g-frame for $U$ with respect to $\left\{V_{\omega}\right\}_{\omega \in \Omega}$. For each $\omega \in \Omega$, let $\Gamma_{\omega} \in \mathscr{B}\left(U, V_{w}\right)$; then, $\Gamma=\left\{\Gamma_{\omega}\right\}_{\omega \in \Omega}$ is a dual continuous $K-g$-Bessel sequence of $\Lambda$ for upper bound $B_{\Gamma}$ if and only if there exists $\varphi \in \mathscr{B}\left(U, \oplus_{\omega \in \Omega} V_{w}\right)$ such that $T_{\Lambda} \varphi=0$ and $\Gamma_{\omega}=\Lambda_{\omega}\left(K^{+}\right)^{*}+P_{\omega} \varphi$ for each $\omega \in \Omega$. In this case, the continuous $g$-Bessel bound of $\Gamma$ is $\left(\left\|K^{+} K\right\|+\|\varphi\|\right)^{2}$. 
Proof. First, assume that $\Gamma$ is a dual continuous $K$-g-Bessel sequence of $\Lambda$, and we define $\varphi: U \longrightarrow \oplus_{\omega \in \Omega} V_{w}$ by $(\varphi x)_{\omega}=$ $\Gamma_{\omega} x-\Lambda_{\omega}\left(K^{+}\right)^{*} x$ for each $x \in U$ and $\omega \in \Omega$; then, $\varphi \in \mathscr{B}\left(U, \oplus_{\omega \in \Omega} V_{w}\right)$. Indeed,

$$
\begin{aligned}
\|\varphi x\| & =\left\|\left\{(\varphi x)_{\omega}\right\}_{\omega \in \Omega}\right\|=\left\|\left\{\Gamma_{\omega} x\right\}_{\omega \in \Omega}-\left\{\Lambda_{\omega}\left(K^{+}\right)^{*} x\right\}_{\omega \in \Omega}\right\| \\
& \leq\left\|\left\{\Gamma_{\omega} x\right\}_{\omega \in \Omega}\right\|+\left\|\left\{\Lambda_{\omega}\left(K^{+}\right)^{*} x\right\}_{\omega \in \Omega}\right\| \\
& =\left(\int_{\Omega}\left\|\Gamma_{\omega} x\right\|^{2}\right)^{(1 / 2)}+\left(\int_{\Omega}\left\|\Lambda_{\omega}\left(K^{+}\right)^{*} x\right\|^{2}\right)^{(1 / 2)} \\
& \leq \sqrt{B_{\Gamma}}\|x\|+\left\|K^{*}\left(K^{+}\right)^{*} x\right\| \\
& \leq\left(\sqrt{B_{\Gamma}}+\|K\|\left\|K^{+}\right\|\right)\|x\|,
\end{aligned}
$$

for each $x \in U$, and by Theorem 1 , we have

$$
\begin{aligned}
T_{\Lambda} \varphi x & =\int_{\Omega} \Lambda_{\omega}^{*}(\varphi x)_{\omega} \mathrm{d} \mu(\omega)=\int_{\Omega} \Lambda_{\omega}^{*}\left(\Gamma_{\omega}-\Lambda_{\omega}\left(K^{+}\right)^{*}\right) x \mathrm{~d} \mu(\omega) \\
& =\int_{\Omega} \Lambda_{\omega}^{*} \Gamma_{\omega} x \mathrm{~d} \mu(\omega)-\int_{\Omega} \Lambda_{\omega}^{*} \Lambda_{\omega}\left(K^{+}\right)^{*} x \mathrm{~d} \mu(\omega)=K x-K x=0 .
\end{aligned}
$$

Conversely, we show that $\left\{\Gamma_{\omega}\right\}_{\omega \in \Omega}$ is a continuous g-Bessel sequence for $U$ with respect to $\left\{V_{\omega}\right\}_{\omega \in \Omega}$ with bound $\left(\left\|K^{+} K\right\|+\|\varphi\|\right)^{2}$.

From Theorem 1, we have

$$
\begin{aligned}
\int_{\Omega} \Lambda_{\omega}^{*} \Gamma_{\omega} x \mathrm{~d} \mu(\omega) & =\int_{\Omega} \Lambda_{\omega}^{*}\left(\Lambda_{\omega}\left(K^{+}\right)^{*}+P_{\omega} \varphi\right) x \mathrm{~d} \mu(\omega) \\
& =\int_{\Omega} \Lambda_{\omega}^{*}\left(\Lambda_{\omega} K^{+}\right)^{*} x \mathrm{~d} \mu(\omega)+T_{\Lambda} \varphi x \\
& =\int_{\Omega} \Lambda_{\omega}^{*}\left(\Lambda_{\omega} K^{+}\right)^{*} x \mathrm{~d} \mu(\omega)=K x, \quad \forall x \in U .
\end{aligned}
$$

Corollary 1. Let $K \in \mathscr{B}(U)$ have a closed range and $\left\{\Lambda_{\omega}\right\}_{\omega \in \Omega}$ be a Parseval continuous $K$-g-frame for $U$ with respect to $\left\{V_{\omega}\right\}_{\omega \in \Omega}$, and let $\Gamma_{\omega} \in \mathscr{B}\left(U, \otimes_{\omega \in \Omega} V_{w}\right), \forall \omega \in \Omega$; then, $\left\{\Gamma_{\omega}\right\}_{\omega \in \Omega}$ is a dual continuous $K$-g-Bessel sequence of $\Lambda$ if and only if there exists a continuous $g$-Bessel sequence $\left\{\theta_{\omega}\right\}_{\omega \in \Omega}$ for $U$ with respect to $\left\{V_{\omega}\right\}_{\omega \in \Omega}$ such that

$$
\begin{gathered}
\Gamma_{\omega}=\Lambda_{\omega}\left(K^{+}\right)^{*}+\theta_{\omega}, \quad \forall \omega \in \Omega, \\
\int_{\Omega} \Lambda_{\omega}^{*} \theta_{\omega} x \mathrm{~d} \mu(\omega)=0, \quad \forall x \in U .
\end{gathered}
$$

Proof. Assume that $\left\{\Gamma_{\omega}\right\}_{\omega \in \Omega}$ is a dual continuous $K$-g-Bessel sequence of $\left\{\Lambda_{\omega}\right\}_{\omega \in \Omega}$; then, from Theorem 2, there exists $\varphi \in \mathscr{B}\left(U, \oplus_{\omega \in \Omega} V_{w}\right)$ such that

$$
\begin{gathered}
\Gamma_{\omega}=\Lambda_{\omega}\left(K^{+}\right)^{*}+P_{\omega} \varphi, \quad \forall \omega \in \Omega, \\
\int_{\Omega} \Lambda_{\omega}^{*}(\varphi x)_{\omega} x=0, \quad \forall x \in U .
\end{gathered}
$$

Taking $\theta_{\omega}=P_{\omega} \varphi, \forall \omega \in \Omega$, then clearly, $\left\{\theta_{\omega}\right\}_{\omega \in \Omega}$ is a continuous g-Bessel sequence for $U$ with respect to $\left\{V_{\omega}\right\}_{\omega \in \Omega}$ with bound $\|\varphi\|^{2}$, and

$$
\int_{\Omega} \Lambda_{\omega}^{*} \theta_{\omega} x \mathrm{~d} \mu(\omega)=\int_{\Omega} \Lambda_{\omega}^{*}(\varphi x)_{\omega} x \mathrm{~d} \mu(\omega), \quad \forall x \in U .
$$

Conversely, let $\left\{\Gamma_{\omega}\right\}_{\omega \in \Omega}=\left\{\Lambda_{\omega}\left(K^{+}\right)^{*}+\theta_{\omega}\right\}_{\omega \in \Omega}$, where $\left\{\theta_{\omega}\right\}_{\omega \in \Omega}$ is a continuous g-Bessel sequence for $U$ with respect to $\left\{V_{\omega}\right\}_{\omega \in \Omega}$ such that

$$
\int_{\Omega} \Lambda_{\omega}^{*} \theta_{\omega} x \mathrm{~d} \mu(\omega)=0
$$

By Theorem 1,

$$
\begin{aligned}
& \int_{\Omega} \Lambda_{\omega}^{*} \Gamma_{\omega} x \mathrm{~d} \mu(\omega) \\
& =\int_{\Omega} \Lambda_{\omega}^{*}\left(\Lambda_{\omega}\left(K^{+}\right)^{*}+\theta_{\omega}\right) x \mathrm{~d} \mu(\omega) \\
& =\int_{\Omega} \Lambda_{\omega}^{*}\left(\Lambda_{\omega}\left(K^{+}\right)^{*}\right) x \mathrm{~d} \mu(\omega)+\int_{\Omega} \Lambda_{\omega}^{*} \theta_{\omega} x \mathrm{~d} \mu(\omega) \\
& =\int_{\Omega} \Lambda_{\omega}^{*}\left(\Lambda_{\omega}\left(K^{+}\right)^{*}\right) x \mathrm{~d} \mu(\omega)=K x .
\end{aligned}
$$

Theorem 3. Let $K \in \mathscr{B}(U)$ have a closed range and $\Lambda=$ $\left\{\Lambda_{\omega}\right\}_{\omega \in \Omega}$ be a Parseval continuous K-g-frame for $U$ with respect to $\left\{V_{\omega}\right\}_{\omega \in \Omega}$; then, $\left\{\Lambda_{\omega}\left(K^{+}\right)^{*}\right\}$ is the canonical dual continuous $K-g$-Bessel sequence of $\Lambda$.

Proof. By Theorem 1, we know that $\left\{\Lambda_{\omega}\left(K^{+}\right)^{*}\right\}$ is a dual continuous $K$-g-Bessel sequence of $\Lambda$; to complete this proof, we need to prove by Proposition 3 that $\left\|T_{\Lambda^{\prime}}\right\| \leq\left\|T_{\Gamma}\right\|$ for any dual continuous $K$-g-Bessel sequence $\left\{\Gamma_{\omega}\right\}_{\omega \in \Omega}$ of $\Lambda$, where $T_{\Lambda^{\prime}}$ is the synthesis operator of $\left\{\Lambda_{\omega}\left(K^{+}\right)^{*}\right\}$. By Theorem 2, there exists $\varphi \in \mathscr{B}\left(U, \oplus_{\omega \in \Omega} V_{w}\right)$ such that $T_{\Lambda} \varphi=0$ and $\Gamma_{\omega}=\Lambda_{\omega}\left(K^{+}\right)^{*}+P_{\omega} \varphi$. A simple computation gives $T_{\Gamma}^{*}=T_{\Lambda^{\prime}}^{*}+\varphi$, noting $T_{\Lambda^{\prime}}=K^{+} T_{\Lambda}$.

For each $x \in U$, we have

$$
\begin{aligned}
\left\|T_{\Gamma}^{*} x\right\|^{2} & =\left\langle T_{\Gamma}^{*} x, T_{\Gamma}^{*} x\right\rangle=\left\langle T_{\Lambda^{\prime}}^{*} x+\varphi x, T_{\Lambda^{\prime}}^{*} x+\varphi x\right\rangle \\
& =\left\|T_{\Lambda^{\prime}}^{*} x\right\|^{2}+\left\langle T_{\Lambda^{\prime}}^{*} x, \varphi x\right\rangle+\left\langle\varphi x, T_{\Lambda^{\prime}}^{*} x\right\rangle+\|\varphi x\|^{2} \\
& =\left\|T_{\Lambda^{\prime}}^{*} x\right\|^{2}+\|\varphi x\|^{2} \geq\left\|T_{\Lambda^{\prime}}^{*} x\right\|^{2} .
\end{aligned}
$$

Therefore, $\left\|T_{\Lambda^{\prime}} x\right\|=\left\|T_{\Lambda^{\prime}}^{*} x\right\| \leq\left\|T_{\Gamma}^{*} x\right\|=\left\|T_{\Gamma} x\right\|$.

The question of stability plays an important role in various fields of applied mathematics. The classical theorem of the stability of a base is due to Paley and Wiener. It is based on the fact that a bounded operator $T$ on a Banach space is invertible if we have $\|I-T\|<1$.

Lemma 3 (see [9]; Paley-Wiener). Let $\left\{f_{i}\right\}_{i \in \mathbb{N}}$ be a basis of a Banach space $X$ and $\left\{g_{i}\right\}_{i \in \mathbb{N}}$ a sequence of vectors in $X$. If there exists a constant $\lambda \in[0,1)$ such that 


$$
\left\|\sum_{i \in \mathbb{N}} c_{i}\left(f_{i}-g_{i}\right)\right\| \leq \lambda\left\|\sum_{i \in \mathbb{N}} c_{i} f_{i}\right\|
$$

for all finite sequences $\left\{c_{i}\right\}_{i \in \mathbb{N}}$ of scalars, then $\left\{g_{i}\right\}_{i \in \mathbb{N}}$ is also a basis for $X$.

Proposition 5. Let $K \in \mathscr{B}(U)$ have a closed range, $\left\{\Lambda_{\omega}\right\}_{\omega \in \Omega}$ be a continuous $K$ - $g$-frame for $U$ with respect to $\left\{V_{\omega}\right\}_{\omega \in \Omega}$ with bounds $A_{\Lambda}$ and $B_{\Lambda}$, and $\left\{\Gamma_{\omega}\right\}_{\omega \in \Omega}$ be a dual continuous $K-g$ Bessel sequence of $\Lambda$ with upper bound $B_{\Lambda}$. For each $\omega \in \Omega$, let $\theta_{\omega} \in B\left(U, V_{\omega}\right)$; if the following conditions are satisfied,

$$
\begin{aligned}
& \text { (1) } \lambda=\int_{\Omega}\left\|\theta_{\omega}-\Lambda_{\omega}\right\|^{2} d \mu(\omega)<\infty \text { and } \\
& \text { (2) } \beta=\int_{\Omega}\left\|K^{+}\right\|\left\|\Lambda_{\omega}-\theta_{\omega}\right\|\|\Gamma\| d \mu(\omega)<1,
\end{aligned}
$$

then $\Theta=\left\{\theta_{\omega}\right\}_{\omega \in \Omega}$ is a $P_{L(\mathscr{R}(K))}$ continuous $K$-g-frame for $U$ with respect to $\left\{V_{\omega}\right\}_{\omega \in \Omega}$ with continuous $K$-g-frame bounds $\left(B_{\Gamma}^{-1}\right)\left\|K^{+}\right\|^{-2}\left(1-\beta^{2}\right)$ and $\left(\sqrt{\lambda}+\sqrt{B_{\Lambda}}\right)^{2}$, where $L$ is defined by $L: \mathscr{R}(K) \longrightarrow U$ such that $L x=\int_{\omega} \theta_{\omega}^{*} \Gamma_{\omega} K^{+} x d \mu(\omega)$ and $P_{L(\mathscr{R}(K))}$ denotes the orthogonal projection on $L(\mathscr{R}(K))$.

Proof. Define

$$
\psi: \underset{\omega \in \Omega}{\oplus} V_{w} \longrightarrow U, \psi\left(\left\{y_{\omega}\right\}_{\omega \in \Omega}\right)=\int_{\omega} \theta_{\omega}^{*} y_{\omega} \mathrm{d} \mu(\omega) .
$$

Then, (1) implies that $\psi$ is well defined and bounded with $\|\psi\| \leq \sqrt{\lambda}+\sqrt{B_{\Lambda}}$.

Clearly, $\psi$ is linear.

Thus, $\int_{\omega} \theta_{\omega}^{*} y_{\omega} \mathrm{d} \mu(\omega)=\left\|\psi^{*}(x)\right\|^{2} \leq\|\psi\|^{2}\|x\|^{2} \leq\left(\sqrt{\lambda}+\sqrt{B_{\Lambda}}\right)^{2}\|x\|^{2}$.

Therefore, $\left\{\theta_{\omega}\right\}_{\omega \in \Omega}$ is a continuous $K$-g-Bessel sequence for $U$ with respect to $\left\{V_{\omega}\right\}_{\omega \in \Omega}$.

For any $x \in \mathscr{R}(K)$, we have

$$
\begin{aligned}
\|x-L x\| & =\| \int_{\omega} \Lambda_{\omega}^{*} \Gamma_{\omega} K^{+} x \mathrm{~d} \mu(\omega)-\int_{\omega} \theta_{\omega}^{*} \Gamma_{\omega} K^{+} x \mathrm{~d} \mu(\omega) \\
& =\| \int_{\omega}\left(\Lambda_{\omega}^{*}-\theta_{\omega}^{*}\right) \Gamma_{\omega} K^{+} x \mathrm{~d} \mu(\omega) \\
& \leq\left\|\int_{\omega}\right\| K^{+}\|\| \Lambda_{\omega}-\theta_{\omega}\|\| \Gamma_{\omega}\|\| x\|\mathrm{~d} \mu(\omega)=\beta\| x \| .
\end{aligned}
$$

Hence,

$$
(1-\beta)\|x\| \leq\|L x\|, \quad \forall x \in \mathscr{R}(K) .
$$

From this, we conclude that the operator $L: \mathscr{R}(K) \longrightarrow L(\mathscr{R}(K))$ is invertible with $\left\|L^{-1}\right\| \leq(1 / 1-\beta)$. It is trivial to show that $L(\mathscr{R}(K))$ is closed.

For any $x \in U$, we have

$$
P_{L(\mathscr{R}(K))} K x=L L^{-1} P_{L(\mathscr{R}(K))} K x=\int_{\Omega} \theta_{\omega}^{*} \Gamma_{\omega} K^{+} L^{-1} P_{L(\mathscr{R}(K))} K x \mathrm{~d} \mu(\omega) .
$$

Therefore,

$$
\begin{aligned}
\left\|K^{*} P_{L(\mathscr{R}(K))} x\right\|^{2} & =\sup _{\|y\|=1}\left|\left\langle K^{*} P_{L(\mathscr{R}(K))} x, y\right\rangle\right|^{2} \\
& =\sup _{\|y\|=1}\left|\int_{\omega}\left\langle\theta_{\omega}^{*} \Gamma_{\omega} K^{+} L^{-1} P_{L(\mathscr{R}(K))} K x, y\right\rangle\right|^{2} \\
& =\sup _{\|y\|=1}\left|\int_{\Omega}\left\langle\Gamma_{\omega} K^{+} L^{-1} P_{L(\mathscr{R}(K))} K x, \theta_{\omega} y\right\rangle\right|^{2} \\
& \leq \int_{\Omega}\left\|\theta_{\omega} x\right\|^{2} \mathrm{~d} \mu(\omega) \sup _{\|y\|=1} \int_{\Omega}\left\|\Gamma_{\omega} K^{+} L^{-1} P_{L(\mathscr{R}(K))} K y\right\|^{2} \mathrm{~d} \mu(\omega) n \\
& \leq B_{\Gamma}\left\|K^{+}\right\|^{2}\left\|L^{-1}\right\|\|K\|^{2} \int_{\Omega}\left\|\theta_{\omega} x\right\|^{2} \mathrm{~d} \mu(\omega) n \\
& \leq B_{\Gamma}\left\|K^{+}\right\|^{2}\|K\|^{2} \frac{1}{(1-\beta)^{2}}\left\|\int_{\Omega}\right\| \theta_{\omega} x \|^{2} \mathrm{~d} \mu(\omega)
\end{aligned}
$$

It follows that

$$
B_{\Gamma}^{-1}\left\|K^{+}\right\|^{-2}\|K\|^{-2}\left\|K^{*} P_{L(\mathscr{R}(K))} x\right\|^{2} \leq \int_{\Omega}\left\|\theta_{\omega} x\right\|^{2} \mathrm{~d} \mu(\omega) .
$$

This completes the proof.

\section{Continuous $K$-g-Frame Sequences and Continuous g-Frame Sequences}

Theorem 4. Let $\Lambda=\left\{\Lambda_{\omega}\right\}_{\omega \in \Omega}$ be a g-frame for $U$ with respect to $\left\{V_{\omega}\right\}_{\omega \in \Omega}$; then, $\Lambda$ is a continuous $K$-g-frame for $U$ with respect to $\left\{V_{\omega}\right\}_{\omega \in \Omega}$ if and only if $\mathscr{R}(K) \subset \overline{\operatorname{span}}\left(\left\{\left\{\Lambda_{\omega}^{*}\left(V_{\omega}\right)\right\}_{\omega \in \Omega}\right)\right.$. 
Proof. Suppose that $\Lambda$ is a continuous $K$-g-frame for $U$ with respect to $\left\{V_{\omega}\right\}_{\omega \in \Omega}$; then, $\forall x \in U$, we have

$$
\begin{aligned}
C_{\Lambda}\left\langle K K^{*} x, x\right\rangle & =C_{\Lambda}\left\|K^{*} x\right\|^{2} \\
& \leq \int_{\Omega}\left\|\Lambda_{\omega} x\right\| \mathrm{d} \mu(\omega)=\left\|T_{\Lambda}^{*} x\right\|^{2} \\
& =\left\langle T_{\Lambda} T_{\Lambda}^{*} x, x\right\rangle .
\end{aligned}
$$

From Lemma 2, we have $\mathscr{R}(K) \subset \mathscr{R}\left(T_{\Lambda}\right)$ since $\mathscr{R}\left(T_{\Lambda}\right) \subseteq \overline{\operatorname{span}}\left(\left\{\Lambda_{\omega}^{*}\left(V_{\omega}\right)\right\}_{\omega \in \Omega}\right) . \quad$ Conversely, let $\mathscr{R}(K) \subset \overline{\operatorname{span}}\left(\left\{\Lambda_{\omega}^{*}\left(V_{\omega}\right)\right\}_{\omega \in \Omega}\right)$.

Since

$U=\overline{\operatorname{span}}\left(\left\{\Lambda_{\omega}^{*}\left(V_{\omega}\right)\right\}_{\omega \in \Omega}\right) \oplus\left(\overline{\operatorname{span}}\left(\left\{\Lambda_{\omega}^{*}\left(V_{\omega}\right)\right\}_{\omega \in \Omega}\right)\right)^{\perp}, \quad$ we have

$$
\begin{aligned}
\forall x & \in U, \\
x & =y+z ; \\
y & \in \overline{\operatorname{span}}\left(\left\{\Lambda_{\omega}^{*}\left(V_{\omega}\right)\right\}_{\omega \in \Omega}\right), \\
z & \left.\in \overline{\operatorname{span}}\left(\left\{\Lambda_{\omega}^{*}\left(V_{\omega}\right)\right\}_{\omega \in \Omega}\right)\right)^{\perp} .
\end{aligned}
$$

For any $\omega \in \Omega, \Lambda_{\omega}^{*}\left(\Lambda_{\omega} z\right) \in \overline{\operatorname{span}}\left(\left\{\Lambda_{\omega}^{*}\left(V_{\omega}\right)\right\}_{\omega \in \Omega}\right)$.

For all $x \in U,\left\|\Lambda_{\omega} x\right\|^{2}=\left\|\Lambda_{\omega} y\right\|^{2}$, so

$\int_{\Omega}\left\|\Lambda_{\omega} x\right\|^{2} \mathrm{~d} \mu(\omega)=\int_{\Omega}\left\|\Lambda_{\omega} y\right\|^{2} \mathrm{~d} \mu(\omega) \leq B_{\Lambda}\left(\|y\|^{2}+\|z\|^{2}\right)=B_{\Lambda}\|x\|^{2}$.

On the contrary, since $\mathscr{R}(K) \subset \overline{\operatorname{span}}\left(\left\{\Lambda_{\omega}^{*}\left(V_{\omega}\right)\right\}_{\omega \in \Omega}\right)$, $z \in(\mathscr{R}(K))^{\perp}$; then, $\left\langle K^{*} z, h\right\rangle=\langle z, K h\rangle=0, h \in U$; hence, $K^{*} z=0$.

Then,

$$
\int_{\Omega}\left\|\Lambda_{\omega} x\right\|^{2} \mathrm{~d} \mu(\omega)=\int_{\Omega}\left\|\Lambda_{\omega} y\right\|^{2} \mathrm{~d} \mu(\omega) \geq C_{\Lambda}\left\|K^{*} y\right\|^{2}=C_{\Lambda}\left\|K^{*} x\right\|^{2} .
$$

Proposition 6. Let $K \in \mathscr{B}(U)$ have a closed range and $\Lambda=$ $\left\{\Lambda_{\omega}\right\}_{\omega \in \Omega}$ be a continuous $K$-g-frame for $U$ with respect to $\left\{V_{\omega}\right\}_{\omega \in \Omega}$; then, $\Lambda=\left\{\Lambda_{\omega}\right\}_{\omega \in \Omega}$ is a continuous $g$-frame for $\mathscr{R}(K)$ with respect to $\left\{V_{\omega}\right\}_{\omega \in \Omega}$.

Proof. We just prove the lower continuous g-frame inequality. From Proposition 2, there exists a continuous g-Bessel sequence $\Gamma=\left\{\Gamma_{\omega}\right\}_{\omega \in \Omega}$ for $U$ with respect to $\left\{V_{\omega}\right\}_{\omega \in \Omega}$ such that $K x=\int_{\Omega} \Lambda_{\omega}^{*} \Gamma_{\omega} x, \forall x \in U$.

For every $y \in \mathscr{R}(K)$, by Lemma 1 ,

$$
y=K K^{+} y=\int_{\Omega} \Lambda_{\omega}^{*} \Gamma_{\omega} K^{+} y \mathrm{~d} \mu(\omega)=\int_{\Omega} \Lambda_{\omega}^{*}\left(\Gamma_{\omega} K^{+} P_{\mathscr{R}(K)}\right) y \mathrm{~d} \mu(\omega) .
$$

Take $\theta_{\omega}=\Gamma_{\omega} K^{+} P_{\mathscr{R}(K)}$ for every $\omega \in \Omega$.

Then, we have

$$
\int_{\Omega}\left\|\theta_{\omega} x\right\|^{2} \mathrm{~d} \mu(\omega)=\int_{\Omega}\left\|\Gamma_{\omega} K^{+} P_{\mathscr{R}(K)}\right\|^{2} \mathrm{~d} \mu(\omega) \leq B_{\Gamma}\left\|K^{+}\right\|^{2}\|x\|^{2} .
$$

So, $\left\{\theta_{\omega}\right\}_{\omega \in \Omega}$ is a continuous g-Bessel sequence for $U$ with respect to $\left\{V_{\omega}\right\}_{\omega \in \Omega}$.

For any $y, z \in \mathscr{R}(K)$, we have

$$
\begin{aligned}
\langle y, z\rangle & =\left\langle\int_{\Omega} \Lambda_{\omega}^{*} \theta_{\omega} y \mathrm{~d} \mu(\omega), z\right\rangle \\
& =\int_{\Omega}\left\langle\Lambda_{\omega}^{*} \theta_{\omega} y, z\right\rangle \mathrm{d} \mu(\omega) \\
& =\int_{\Omega}\left\langle\Gamma_{\omega} K^{+} P_{\mathscr{R}(K)} y, \Lambda_{\omega} z\right\rangle \mathrm{d} \mu(\omega) \\
& =\int_{\Omega}\left\langle y,\left(\Gamma_{\omega} K^{+} P_{\mathscr{R}(K)}\right)^{*}, \Lambda_{\omega} z\right\rangle \mathrm{d} \mu(\omega) \\
& =\left\langle y, \int_{\Omega}\left(\Gamma_{\omega} K^{+} P_{\mathscr{R}(K)}\right)^{*} \Lambda_{\omega} z\right\rangle \mathrm{d} \mu(\omega) \\
& =\left\langle y, \int_{\Omega} \theta_{\omega} \Lambda_{\omega} z \mathrm{~d} \mu(\omega)\right\rangle,
\end{aligned}
$$

and then $z=\int_{\Omega} \theta_{\omega} \Lambda_{\omega} z \mathrm{~d} \mu(\omega)$.

Hence,

$$
\begin{aligned}
\|z\|^{2} & =\sup _{\|y\|=1}|\langle z, y\rangle|^{2} \\
& =\sup _{\|y\|=1}\left|\left\langle\int_{\Omega} \theta_{\omega} \Lambda_{\omega} z \mathrm{~d} \mu(\omega), y\right\rangle\right|^{2} \\
& \leq\left(\sup _{\|y\|=1} \int_{\Omega}\left\|\theta_{\omega} y\right\|^{2} \mathrm{~d} \mu(\omega)\right)\left(\int_{\Omega}\left\|\Lambda_{\omega} z\right\|^{2} \mathrm{~d} \mu(\omega)\right) \\
& \leq B_{\Gamma}\left\|K^{+}\right\|^{2}\left(\int_{\Omega}\left\|\Lambda_{\omega} z\right\|^{2} \mathrm{~d} \mu(\omega)\right) .
\end{aligned}
$$

Then, $\left\{\Lambda_{\omega}\right\}_{\omega \in \Omega}$ is a continuous $g$-frame for $\mathscr{R}(K)$ with respect to $\left\{V_{\omega}\right\}_{\omega \in \Omega}$.

Proposition 7. Let $K \in \mathscr{B}(U)$ have a closed range and $\Lambda=$ $\left\{\Lambda_{\omega}\right\}_{\omega \in \Omega}$ be a continuous $K$-g-frame for $\mathscr{R}(K)$ with respect to $\left\{V_{\omega}\right\}_{\omega \in \Omega}$. If $\overline{\operatorname{span}}\left(\left\{\Lambda_{\omega}^{*}\left(V_{\omega}\right)\right\}_{\omega \in \Omega}\right) \subset \mathscr{R}(K)$, then $\Lambda=\left\{\Lambda_{\omega}\right\}_{\omega \in \Omega}$ is a continuous $K$-g-frame for $U$ with respect to $\left\{V_{\omega}\right\}_{\omega \in \Omega}$.

Proof. For each $x \in U$, we have $x=y+z$ such that $y \in \mathscr{R}(K)$ and $z \in(\mathscr{R}(K))^{\perp}$. So, $\left\|\Lambda_{\omega} x\right\|^{2}=\left\|\Lambda_{\omega} y\right\|^{2}$.

Since $\overline{\operatorname{span}}\left(\left\{\Lambda_{\omega}^{*}\left(V_{\omega}\right)\right\}_{\omega \in \Omega}\right) \subset \mathscr{R}(K)$ and $\Lambda_{\omega}^{*}\left(\Lambda_{\omega} e\right) \in \overline{\operatorname{span}}\left(\left\{\Lambda_{\omega}^{*}\left(V_{\omega}\right)\right\}_{\omega \in \Omega}\right), \forall e \in U$,

$$
\int_{\Omega}\|\Lambda x\|^{2} \mathrm{~d} \mu(\omega)=\int_{\Omega}\|\Lambda y\|^{2} \mathrm{~d} \mu(\omega) \leq B_{\Lambda}\|x\|^{2} \leq B_{\Lambda}\left(\|y\|^{2}+\|z\|^{2}\right)=B_{\Lambda}\|x\|^{2} .
$$


Let $\Phi=\left\{\phi_{\omega}\right\}_{\omega \in \Omega}$ be a dual continuous $\mathrm{g}$-frame of $\Lambda=\left\{\Lambda_{\omega}\right\}_{\omega \in \Omega}$.

So,

$$
\int_{\Omega} \Lambda_{\omega}^{*} \phi_{\omega} y \mathrm{~d} \mu(\omega)=\int_{\Omega} \phi_{\omega}^{*} \Lambda_{\omega} y \mathrm{~d} \mu(\omega) .
$$

Hence,

$$
\begin{aligned}
\left\|K^{*} x\right\|^{2} & =\left\|K^{*}(y+z)\right\|=\left\|K^{*} y\right\|^{2}=\left|\left\langle K^{*} y, K^{*} y\right\rangle\right| \\
& =\left|\left\langle K K^{*} y, y\right\rangle\right|=\left|\left\langle\int_{\Omega} \phi_{\omega}^{*} \Lambda_{\omega} y \mathrm{~d} \mu(\omega), K K^{*} y\right\rangle\right| \\
& =\left|\int_{\Omega}\left\langle\Lambda_{\omega} y, \phi_{\omega} K K^{*} y\right\rangle \mathrm{d} \mu(\omega)\right| \\
& \leq\left(\int_{\Omega}\left\|\Lambda_{\omega} y\right\|^{2} \mathrm{~d} \mu(\omega)\right)^{(1 / 2)}\left(\int_{\Omega}\left\|\phi_{\omega} K K^{*} y\right\|^{2} \mathrm{~d} \mu(\omega)\right)^{(1 / 2)} \\
& \leq \sqrt{B_{\phi}}\left\|K K^{*} y\right\|\left(\int_{\Omega}\left\|\Lambda_{\omega} y\right\|^{2}\right)^{(1 / 2)} \\
& \leq \sqrt{B_{\phi}}\|K\|\left\|K^{*} x\right\|\left(\int_{\Omega}\left\|\Lambda_{\omega} y\right\|^{2} \mathrm{~d} \mu(\omega)\right)^{(1 / 2)}
\end{aligned}
$$

Then, $B_{\phi}^{-1}\|K\|^{-2}\left\|K^{*} x\right\|^{2} \leq \int_{Q}\left\|\Lambda_{\omega} y\right\|^{2}$.

On the contrary, we have $\int_{\Omega}\left\|\Lambda_{\omega} y\right\|^{2}=\int_{\Omega}\left\|\Lambda_{\omega} x\right\|^{2}$.

Then, $B_{\phi}^{-1}\|K\|^{-2}\left\|K^{*} x\right\|^{2} \leq \int_{\Omega}\left\|\Lambda_{\omega} x\right\|^{2}$.

So, $\Lambda$ is a continuous $K$-g-frame for $U$.

Theorem 5. For every $\omega \in \Omega$, let $\Lambda_{\omega} \in \mathscr{B}\left(U, V_{w}\right)$; then, the following statements are equivalent:

(1) $\Lambda=\left\{\Lambda_{\omega}\right\}_{\omega \in \Omega}$ is a continuous $K$ - $g$-frame sequence for $U$ with respect to $\left\{V_{\omega}\right\}_{\omega \in \Omega}$.

(2) $\Lambda=\left\{\Lambda_{\omega}\right\}_{\omega \in \Omega}$ is a continuous $g$-Bessel sequence for $\overline{\operatorname{span}}\left(\left\{\Lambda_{\omega}^{*}\left(V_{\omega}\right)\right\}_{\omega \in \Omega}\right)$ with respect to $\left\{V_{\omega}\right\}_{\omega \in \Omega}$, and there exists a continuous $g$-Bessel sequence $\left\{\Gamma_{\omega}\right\}_{\omega \in \Omega}$ for $U$ with respect to $\left\{V_{\omega}\right\}_{\omega \in \Omega}$ such that

$$
\left(\frac{K^{*}}{\overline{\operatorname{span}}\left(\left\{\Lambda_{\omega}^{*}\left(V_{\omega}\right)\right\}_{\omega \in \Omega}\right)}\right)^{*} x=\int_{\Omega} \Lambda_{\omega}^{*} \Gamma_{\omega} x, \quad \forall x \in U
$$

(3) $\Lambda=\left\{\Lambda_{\omega}\right\}_{\omega \in \Omega}$ is a continuous $g$-Bessel sequence for $\overline{\operatorname{span}}\left(\left\{\Lambda_{\omega}^{*}\left(V_{\omega}\right)\right\}_{\omega \in \Omega}\right)$ with respect to $\left\{V_{\omega}\right\}_{\omega \in \Omega}$, and there exists a continuous $g$-Bessel sequence $\left\{\Gamma_{\omega}\right\}_{\omega \in \Omega}$ for $U$ with respect to $\left\{V_{\omega}\right\}_{\omega \in \Omega}$ such that

$$
K^{*} x=\int_{\Omega} \Gamma_{\omega}^{*} \Lambda_{\omega} x \mathrm{~d} \mu(\omega), \quad \forall x \in \overline{\operatorname{span}}\left(\left\{\Lambda_{\omega}^{*}\left(V_{\omega}\right)\right\}_{\omega \in \Omega}\right) .
$$

$$
\frac{K^{*}}{\overline{\operatorname{span}}\left(\left\{\Lambda_{\omega}^{*}\left(V_{\omega}\right)\right\}_{\omega \in \Omega}\right)}: \overline{\operatorname{span}}\left(\left\{\Lambda_{\omega}^{*}\left(V_{\omega}\right)\right\}_{\omega \in \Omega}\right) \longrightarrow U
$$

then their adjoint

$\left(\frac{K^{*}}{\overline{\operatorname{span}}\left(\left\{\Lambda_{\omega}^{*}\left(V_{\omega}\right)\right\}_{\omega \in \Omega}\right)}\right)^{*}: U \longrightarrow \overline{\operatorname{span}}\left(\left\{\Lambda_{\omega}^{*}\left(V_{\omega}\right)\right\}_{\omega \in \Omega}\right)$.

By the definition on the continuous $K$-g-frame sequence, there exists $C_{\Lambda}>0$ such that

$$
C_{\Lambda}\left\|K^{*} x\right\|^{2} \leq\left\|T_{\Lambda}^{*} x\right\|^{2}, \quad \forall x \in \overline{\operatorname{span}}\left(\left\{\Lambda_{\omega}^{*}\left(V_{\omega}\right)\right\}_{\omega \in \Omega}\right) .
$$

This implies

$$
C_{\Lambda}\left(\frac{K^{*}}{\overline{\operatorname{span}}\left(\left\{\Lambda_{\omega}^{*}\left(V_{\omega}\right)\right\}_{\omega \in \Omega}\right)}\right)^{*}\left(\frac{K^{*}}{\overline{\operatorname{span}}\left(\left\{\Lambda_{\omega}^{*}\left(V_{\omega}\right)\right\}_{\omega \in \Omega}\right)}\right) \leq T_{\Lambda} T_{\Lambda}^{*} .
$$

From Lemma 2, there exists $R \in \mathscr{B}\left(U, \oplus_{\omega \in \Omega} V_{w}\right)$ such that

$$
\left(\frac{K^{*}}{\overline{\operatorname{span}}\left(\left\{\Lambda_{\omega}^{*}\left(V_{\omega}\right)\right\}_{\omega \in \Omega}\right)}\right)^{*}=T_{\Lambda} R .
$$

For every $\omega \in \Omega$, denote $\Gamma_{\omega}=P_{\omega} R$; then, we have

$$
\begin{aligned}
\int_{\Omega}\left\|\Gamma_{\omega} x\right\|^{2} \mathrm{~d} \mu(\omega) & =\int_{\Omega}\left\|P_{\omega} R x\right\|^{2} \mathrm{~d} \mu(\omega) \\
& =\int_{\Omega}\left\|(R x)_{\omega}\right\|^{2} \mathrm{~d} \mu(\omega) \\
& =\|R x\|^{2} \\
& \leq\|R\|^{2}\|x\|^{2} .
\end{aligned}
$$

Hence, $\left\{\Gamma_{\omega}\right\}_{\omega \in \Omega}$ is a continuous g-Bessel sequence for $U$ with respect to $\left\{V_{\omega}\right\}_{\omega \in \Omega}$.

On the contrary, we have for all $x \in U$,

$$
\begin{aligned}
\left(\frac{K^{*}}{\overline{\operatorname{span}}\left(\left\{\Lambda_{\omega}^{*}\left(V_{\omega}\right)\right\}_{\omega \in \Omega}\right)}\right)^{*} x & =T_{\Lambda} R x \\
& =\int_{\Omega} \Lambda_{\omega}^{*}(R x)_{\omega} \mathrm{d} \mu(\omega) \\
& =\int_{\Omega} \Lambda_{\omega}^{*}\left(P_{\omega} R x\right) \mathrm{d} \mu(\omega) \\
& =\int_{\Omega} \Lambda_{\omega}^{*} \Gamma_{\omega} x \mathrm{~d} \mu(\omega) .
\end{aligned}
$$

Proof. $\quad(1) \Longrightarrow(2)$ :

We have

$(2) \Longrightarrow(3)$ : 
For every $x \in U$ and $y \in \overline{\operatorname{span}}\left(\left\{\Lambda_{\omega}^{*}\left(V_{\omega}\right)\right\}_{\omega \in \Omega}\right)$, by (48),

we have

$$
\left\langle\left(\frac{K^{*}}{\overline{\operatorname{span}}\left(\left\{\Lambda_{\omega}^{*}\left(V_{\omega}\right)\right\}_{\omega \in \Omega}\right)}\right)^{*} x, y\right\rangle=\int_{\Omega}\left\langle\Lambda_{\omega}^{*} \Gamma_{\omega} x, y\right\rangle \mathrm{d} \mu(\omega)=\left\langle x, \int_{\Omega} \Gamma_{\omega}^{*} \Lambda_{\omega} y \mathrm{~d} \mu(\omega)\right\rangle .
$$

So,

$$
\left\langle x, K^{*} y\right\rangle=\left\langle x, \frac{K^{*}}{\operatorname{span}\left(\left\{\Lambda_{\omega}^{*}\left(V_{\omega}\right)\right\}_{\omega \in \Omega}\right) y}\right\rangle=\left\langle x, \int_{\Omega} \Gamma_{\omega}^{*} \Lambda_{\omega} y \mathrm{~d} \mu(\omega)\right\rangle .
$$

Hence, $K^{*} y=\int_{\Omega} \Gamma_{\omega}^{*} \Lambda_{\omega} y \mathrm{~d} \mu(\omega)$.

(3) $\Longrightarrow(1)$ :

Suppose that (49) holds; to prove (1), we just prove the lower bound inequality on continuous $K$-g-frames.

For every $y \in \overline{\operatorname{span}}\left(\left\{\Lambda_{\omega}^{*}\left(V_{\omega}\right)\right\}_{\omega \in \Omega}\right)$, we have

$$
\begin{aligned}
\left\|K^{*} y\right\|^{2} & =\sup _{\|z\|=1}\left|\left\langle K^{*} y, z\right\rangle\right|^{2}=\sup _{\|z\|=1}\left|\left\langle\int_{\Omega} \Gamma_{\omega}^{*} \Lambda_{\omega} y \mathrm{~d} \mu(\omega), z\right\rangle\right|^{2} \\
& =\sup _{\|z\|=1}\left|\left\langle\int_{\Omega} \Lambda_{\omega} y \mathrm{~d} \mu(\omega), \Gamma_{\omega} z\right\rangle\right|^{2} \\
& \leq \int_{\Omega}\left\|\Lambda_{\omega} y\right\|^{2} \mathrm{~d} \mu(\omega) \sup _{\|z\|=1} \int_{\Omega}\left\|\Gamma_{\omega} z\right\|^{2} \mathrm{~d} \mu(\omega) \\
& \leq B_{\Gamma} \int_{\Omega}\left\|\Lambda_{\omega} y\right\|^{2} \mathrm{~d} \mu(\omega) .
\end{aligned}
$$

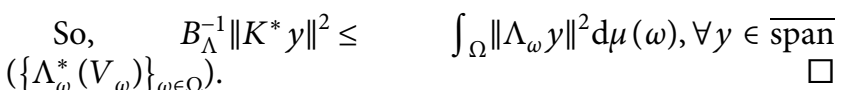

Theorem 6. A sequence $\left\{\Lambda_{\omega}\right\}_{\omega \in \Omega} \in \mathscr{B}\left(U, \oplus_{\omega \in \Omega} V_{w}\right)$ is a continuous $K$ - $g$-frame sequence for $U$ with respect to $\left\{V_{\omega}\right\}_{\omega \in \Omega}$ if and only if there exists $T \in \mathscr{B}\left(\overline{\operatorname{span}}\left(\left\{\Lambda_{\omega}^{*}\left(V_{\omega}\right)\right\}_{\omega \in \Omega}\right), \oplus_{\omega \in \Omega} V_{w}\right)$ such that

$$
\begin{aligned}
& \frac{\Lambda_{\omega}}{\overline{\operatorname{span}}\left(\left\{\Lambda_{\omega}^{*}\left(V_{\omega}\right)\right\}_{\omega \in \Omega}\right)}=P_{\omega} T, \quad \forall \omega \in \Omega, \\
& \mathscr{R}\left(\frac{K^{*}}{\overline{\operatorname{span}}\left(\left\{\Lambda_{\omega}^{*}\left(V_{\omega}\right)\right\}_{\omega \in \Omega}\right)}\right) \subset \mathscr{R}\left(T^{*}\right) .
\end{aligned}
$$

Proof. Let $\left\{\Lambda_{\omega}\right\}_{\omega \in \Omega}$ be a continuous $K$-g-frame sequence for $U$ with respect to $\left\{V_{\omega}\right\}_{\omega \in \Omega}$, and let $T=T_{\Lambda}^{*}$.
Hence, $\Lambda_{\omega} / \overline{\operatorname{span}}\left(\left\{\Lambda_{\omega}^{*}\left(V_{\omega}\right)\right\}_{\omega \in \Omega}\right) x=P_{\omega}\left(T_{\Lambda}^{*} x\right)=P_{\omega}(T x)$ for every $x \in \overline{\operatorname{span}}\left(\left\{\Lambda_{\omega}^{*}\left(V_{\omega}\right)\right\}_{\omega \in \Omega}\right)$ and any $\omega \in \Omega$. So, $\Lambda_{\omega} / \overline{\operatorname{span}}\left(\left\{\Lambda_{\omega}^{*}\left(V_{\omega}\right)\right\}_{\omega \in \Omega}\right)=P_{\omega} T$.

From (53), we have

$$
C_{\Lambda}\left(\frac{K^{*}}{\overline{\operatorname{span}}\left(\left\{\Lambda_{\omega}^{*}\left(V_{\omega}\right)\right\}_{\omega \in \Omega}\right)}\right)^{*}\left(\frac{K^{*}}{\overline{\operatorname{span}}\left(\left\{\Lambda_{\omega}^{*}\left(V_{\omega}\right)\right\}_{\omega \in \Omega}\right)}\right) \leq T^{*} T .
$$

By Lemma 2, it follows that $\mathscr{R}\left(\left(K^{*} / \overline{\operatorname{span}}\left(\left\{\Lambda_{\omega}^{*}\left(V_{\omega}\right)\right\}_{\omega \in \Omega}\right)\right)^{*}\right) \subset \mathscr{R}\left(T^{*}\right)$.

Conversely, $\Lambda_{\omega} / \overline{\operatorname{span}}\left(\left\{\Lambda_{\omega}^{*}\left(V_{\omega}\right)\right\}_{\omega \in \Omega}\right)=P_{\omega} T$ for any $\omega \in \Omega$.

Let $x \in \overline{\operatorname{span}}\left(\left\{\Lambda_{\omega}^{*}\left(V_{\omega}\right)\right\}_{\omega \in \Omega}\right)$; we have

$$
\begin{aligned}
\int_{\Omega}\left\|\Lambda_{\omega} x\right\|^{2} \mathrm{~d} \mu(\omega) & =\int_{\Omega}\left\|\frac{\Lambda_{\omega}}{\overline{\operatorname{span}}\left(\left\{\Lambda_{\omega}^{*}\left(V_{\omega}\right)\right\}_{\omega \in \Omega}\right) x}\right\|^{2} \mathrm{~d} \mu(\omega) \\
& =\int_{\Omega}\left\|P_{\omega} T\right\|^{2} \mathrm{~d} \mu(\omega) \\
& =\|T x\|^{2} \\
& \leq\|T\|^{2}\|x\|^{2} .
\end{aligned}
$$

So, $\left\{\Lambda_{\omega}\right\}_{\omega \in \Omega}$ is a continuous $K$-g-frame sequence for $\overline{\operatorname{span}}\left(\left\{\Lambda_{\omega}^{*}\left(V_{\omega}\right)\right\}_{\omega \in \Omega}\right)$ with respect to $\left\{V_{\omega}\right\}_{\omega \in \Omega}$.

Since $\mathscr{R}\left(\left(K^{*} / \overline{\operatorname{span}}\left(\left\{\Lambda_{\omega}^{*}\left(V_{\omega}\right)\right\}_{\omega \in \Omega}\right)\right)^{*}\right) \subset \mathscr{R}\left(T^{*}\right)$, by Lemma 2, there exists $\lambda>0$ such that

$$
\left(\left(\frac{K^{*}}{\overline{\operatorname{span}}\left(\left\{\Lambda_{\omega}^{*}\left(V_{\omega}\right)\right\}_{\omega \in \Omega}\right)}\right)^{*}\right) \frac{K^{*}}{\overline{\operatorname{span}}\left(\left\{\Lambda_{\omega}^{*}\left(V_{\omega}\right)\right\}_{\omega \in \Omega}\right)} \leq \lambda T^{*} T .
$$

Hence, for every $x \in \overline{\operatorname{span}}\left(\left\{\Lambda_{\omega}^{*}\left(V_{\omega}\right)\right\}_{\omega \in \Omega}\right)$, we have

$$
\lambda^{-1}\left\|K^{*} x\right\|^{2}=\lambda^{-1}\left\|\left(\frac{K^{*}}{\overline{\operatorname{span}}\left(\left\{\Lambda_{\omega}^{*}\left(V_{\omega}\right)\right\}_{\omega \in \Omega}\right)}\right) x\right\|^{2} \leq\|T x\|^{2}=\int_{\Omega}\|\Lambda x\|^{2} \mathrm{~d} \mu \omega,
$$


and then $\left\{\Lambda_{\omega}\right\}_{\omega \in \Omega}$ is a continuous $K$-g-frame sequence for $\overline{\operatorname{span}}\left(\left\{\Lambda_{\omega}^{*}\left(V_{\omega}\right)\right\}_{\omega \in \Omega}\right)$ with respect to $\left\{V_{\omega}\right\}_{\omega \in \Omega}$ with bounds $\lambda^{-1}$ and $\|T\|^{2}$.

In general, a continuous $K$-g-frame $\left\{\Lambda_{\omega}\right\}_{\omega \in \Omega}$ for $U$ with respect to $\left\{V_{\omega}\right\}_{\omega \in \Omega}$ is not a continuous g-frame for $\overline{\operatorname{span}}\left(\left\{\Lambda_{\omega}^{*}\left(V_{\omega}\right)\right\}_{\omega \in \Omega}\right)$ with respect to $\left\{V_{\omega}\right\}_{\omega \in \Omega}$.

Proposition 8. Let $\Lambda=\left\{\Lambda_{\omega}\right\}_{\omega \in \Omega}$ be a continuous $K$-g-frame sequence for $U$ with respect to $\left\{V_{\omega}\right\}_{\omega \in \Omega}$. Suppose that $K^{*} / \overline{\operatorname{span}}\left(\left\{\Lambda_{\omega}^{*}\left(V_{\omega}\right)\right\}_{\omega \in \Omega}\right) \neq 0$ and that it has a closed range; then, $\left\{\Lambda_{\omega}\right\}_{\omega \in \Omega}$ is a continuous g-frame for $\mathscr{R}\left(K ^ { * } \longdiv { \operatorname { s p a n } } ( \{ \Lambda _ { \omega } ^ { * } ( V _ { \omega } ) \} _ { \omega \in \Omega } )\right)^{*}$ with respect to $\left\{V_{\omega}\right\}_{\omega \in \Omega}$.

Proof. Since $K^{*} / \overline{\operatorname{span}}\left(\left\{\Lambda_{\omega}^{*}\left(V_{\omega}\right)\right\}_{\omega \in \Omega}\right)$ has a closed range, its pseudo-inverse exists; from Lemma 1, every $x \in \mathscr{R}\left(K^{*} / \overline{\operatorname{span}}\left(\left\{\Lambda_{\omega}^{*}\left(V_{\omega}\right)\right\}_{\omega \in \Omega}\right)\right)^{*}$ can be written as

$$
x=\left(\frac{K^{*}}{\overline{\operatorname{span}}\left(\left\{\Lambda_{\omega}^{*}\left(V_{\omega}\right)\right\}_{\omega \in \Omega}\right)}\right)^{+}\left(\frac{K^{*}}{\overline{\operatorname{span}}\left(\left\{\Lambda_{\omega}^{*}\left(V_{\omega}\right)\right\}_{\omega \in \Omega}\right)}\right) x .
$$

Hence,

$$
\|x\|^{2} \leq\left\|\left(\frac{K^{*}}{\overline{\operatorname{span}}\left(\left\{\Lambda_{\omega}^{*}\left(V_{\omega}\right)\right\}_{\omega \in \Omega}\right)}\right)^{+}\right\|^{2}\left\|\frac{K^{*}}{\overline{\operatorname{span}}\left(\left\{\Lambda_{\omega}^{*}\left(V_{\omega}\right)\right\}_{\omega \in \Omega}\right) x}\right\|^{2} .
$$

Noting that $\mathscr{R}\left(K^{*} / \overline{\operatorname{span}}\left(\left\{\Lambda_{\omega}^{*}\left(V_{\omega}\right)\right\}_{\omega \in \Omega}\right)\right)^{*} \subset \overline{\operatorname{span}}$ $\left(\left\{\Lambda_{\omega}^{*}\left(V_{\omega}\right)\right\}_{\omega \in \Omega}\right)$, we have

$$
\left(\frac{K^{*}}{\overline{\operatorname{span}}\left(\left\{\Lambda_{\omega}^{*}\left(V_{\omega}\right)\right\}_{\omega \in \Omega}\right)}\right) x=K^{*} x .
$$

So,

$$
\begin{aligned}
\|x\|^{2} & \leq\left\|\left(\frac{K^{*}}{\overline{\operatorname{span}}\left(\left\{\Lambda_{\omega}^{*}\left(V_{\omega}\right)\right\}_{\omega \in \Omega}\right)}\right)^{+}\right\|^{2}\left\|K^{*} x\right\|^{2} \\
& \leq \frac{1}{A_{\Lambda}}\left\|\left(\frac{K^{*}}{\overline{\operatorname{span}}\left(\left\{\Lambda_{\omega}^{*}\left(V_{\omega}\right)\right\}_{\omega \in \Omega}\right)}\right)^{+}\right\|^{2} \int_{\Omega}\left\|\Lambda_{\omega} x\right\|^{2} \mathrm{~d} \mu(\omega) .
\end{aligned}
$$

Since $K^{*} / \overline{\operatorname{span}}\left(\left\{\Lambda_{\omega}^{*}\left(V_{\omega}\right)\right\}_{\omega \in \Omega}\right) \neq 0$, its pseudo-inverse

$$
\left(\frac{K^{*}}{\overline{\operatorname{span}}\left(\left\{\Lambda_{\omega}^{*}\left(V_{\omega}\right)\right\}_{\omega \in \Omega}\right)}\right)^{+} \neq 0
$$

and then
$A_{\Lambda}\left\|\left(\frac{K^{*}}{\overline{\operatorname{span}}\left(\left\{\Lambda_{\omega}^{*}\left(V_{\omega}\right)\right\}_{\omega \in \Omega}\right)}\right)^{+}\right\|^{-2}\|x\|^{2} \leq \int_{\Omega}\left\|\Lambda_{\omega} x\right\|^{2} \mathrm{~d} \mu(\omega)$.

On the contrary, we have

$\int_{\Omega}\left\|\Lambda_{\omega} x\right\|^{2} \mathrm{~d} \mu(\omega) \leq B_{\Lambda}\|x\|^{2}, \quad \forall x \in \mathscr{R}\left(\left(\frac{K^{*}}{\overline{\operatorname{span}}\left(\left\{\Lambda_{\omega}^{*}\left(V_{\omega}\right)\right\}_{\omega \in \Omega}\right)}\right)^{*}\right)$.

So, $\left\{\Lambda_{\omega}\right\}_{\omega \in \Omega}$ is a continuous g-frame for $\mathscr{R}\left(\left(K^{*} / \overline{\operatorname{span}}\left(\left\{\Lambda_{\omega}^{*}\left(V_{\omega}\right)\right\}_{\omega \in \Omega}\right)\right)^{*}\right)$ with respect to $\left\{V_{\omega}\right\}_{\omega \in \Omega}$ with bounds $C_{\Lambda}\left\|\left(K^{*} / \operatorname{span}\left(\left\{\Lambda_{\omega}^{*}\left(V_{\omega}\right)\right\}_{\omega \in \Omega}\right)\right)^{+}\right\|^{-2}$ and $B_{\Lambda}$.

In the following, we give a necessary and sufficient condition under which a tight continuous $K$-g-frame sequence $\left\{\Lambda_{\omega}\right\}_{\omega \in \Omega}$ for $U$ with respect to $\left\{V_{\omega}\right\}_{\omega \in \Omega}$ is a tight continuous g-frame for $\overline{\operatorname{span}}\left(\left\{\Lambda_{\omega}^{*}\left(V_{\omega}\right)\right\}_{\omega \in \Omega}\right)$ with respect to $\left\{V_{\omega}\right\}_{\omega \in \Omega}$.

Proof. Theorem 7Let $\left\{\Lambda_{\omega}\right\}_{\omega \in \Omega}$ be a $C$-tight continuous g-frame for $U$ with respect to $\left\{V_{\omega}\right\}_{\omega \in \Omega}$; then, $\left\{\Lambda_{\omega}\right\}_{\omega \in \Omega}$ is a $D$-tight continuous g-frame for $\operatorname{span}\left(\left\{\Lambda_{\omega}^{*}\left(V_{\omega}\right)\right\}_{\omega \in \Omega}\right)$ with respect to $\left\{V_{\omega}\right\}_{\omega \in \Omega}$ if and only if $K^{*} / \overline{\operatorname{span}}\left(\left\{\Lambda_{\omega}^{*}\left(V_{\omega}\right)\right\}_{\omega \in \Omega}\right)$ admits a left inverse $C / D\left(K^{*} / \overline{\operatorname{span}}\left(\left\{\Lambda_{\omega}^{*}\left(V_{\omega}\right)\right\}_{\omega \in \Omega}\right)\right)^{*}$.

Proof. First, we suppose that $\left\{\Lambda_{\omega}\right\}_{\omega \in \Omega}$ is a $D$-tight continuous g-frame for $\overline{\operatorname{span}}\left(\left\{\Lambda_{\omega}^{*}\left(V_{\omega}\right)\right\}_{\omega \in \Omega}\right)$ with respect to $\left\{V_{\omega}\right\}_{\omega \in \Omega}$.

Hence,

$$
\begin{aligned}
D\|x\|^{2} & =\int_{\Omega}\left\|\Lambda_{\omega} x\right\|^{2} \mathrm{~d} \mu(\omega), \quad K 4 \forall x \in \overline{\operatorname{span}}\left(\left\{\Lambda_{\omega}^{*}\left(V_{\omega}\right)\right\}_{\omega \in \Omega}\right), \\
C\left\|K^{*} x\right\|^{2} & =\int_{\Omega}\left\|\Lambda_{\omega} x\right\|^{2} \mathrm{~d} \mu(\omega), \quad \forall x \in \overline{\operatorname{span}}\left(\left\{\Lambda_{\omega}^{*}\left(V_{\omega}\right)\right\}_{\omega \in \Omega}\right) .
\end{aligned}
$$
Then,

So, $D\|x\|^{2}=C\left\|K^{*} x\right\|^{2}$ for every $x \in \overline{\operatorname{span}}\left(\left\{\Lambda_{\omega}^{*}\left(V_{\omega}\right)\right\}_{\omega \in \Omega}\right)$.

$$
\begin{aligned}
D\langle x, x\rangle & =C\left\langle K^{*} x, K^{*} x\right\rangle \\
& =C\left\langle\left(\frac{K^{*}}{\overline{\operatorname{span}}\left(\left\{\Lambda_{\omega}^{*}\left(V_{\omega}\right)\right\}_{\omega \in \Omega}\right)}\right)^{*}\left(\frac{K^{*}}{\overline{\operatorname{span}}\left(\left\{\Lambda_{\omega}^{*}\left(V_{\omega}\right)\right\}_{\omega \in \Omega}\right)}\right) x, x\right\rangle .
\end{aligned}
$$

This implies that

$$
\begin{aligned}
& \frac{C}{D}\left(\frac{K}{\overline{\operatorname{span}}\left(\left\{\Lambda_{\omega}^{*}\left(V_{\omega}\right)\right\}_{\omega \in \Omega}\right)}\right)^{*}\left(\frac{K^{*}}{\overline{\operatorname{span}}\left(\left\{\Lambda_{\omega}^{*}\left(V_{\omega}\right)\right\}_{\omega \in \Omega}\right)}\right)=I d_{\overline{\operatorname{span}}}\left(\left\{\Lambda_{\omega}^{*}\left(V_{\omega}\right)\right\}_{\omega \in \Omega}\right) . \\
& x=\frac{C}{D}\left(\frac{K^{*}}{\overline{\operatorname{span}}\left(\left\{\Lambda_{\omega}^{*}\left(V_{\omega}\right)\right\}_{\omega \in \Omega}\right)}\right)^{*} .
\end{aligned}
$$


So,

$$
\begin{aligned}
\int_{\Omega}\left\|\Lambda_{\omega} x\right\|^{2} \mathrm{~d} \mu(\omega) & =C\left\|K^{*} x\right\|^{2}=D \frac{C}{D}\left\|K^{*} x\right\|^{2} \\
& =D \frac{C}{D}\left\langle\left(\frac{K^{*}}{\overline{\operatorname{span}}\left(\left\{\Lambda_{\omega}^{*}\left(V_{\omega}\right)\right\}_{\omega \in \Omega}\right)}\right) x,\left(\frac{K^{*}}{\overline{\operatorname{span}}\left(\left\{\Lambda_{\omega}^{*}\left(V_{\omega}\right)\right\}_{\omega \in \Omega}\right)}\right) x\right\rangle \\
& =D\|x\|^{2} .
\end{aligned}
$$

It follows that $\Lambda=\left\{\Lambda_{\omega}\right\}_{\omega \in \Omega}$ is a $D$-tight continuous g-frame for $\overline{\operatorname{span}}\left(\left\{\Lambda_{\omega}^{*}\left(V_{\omega}\right)\right\}_{\omega \in \Omega}\right)$ with respect to $\left\{V_{\omega}\right\}_{\omega \in \Omega}$.
Theorem 7. Let $K_{1}, K_{2} \in \mathscr{B}(U)$ and $\left\{\Lambda_{\omega}\right\}_{\omega \in \Omega}$ be a C-tight continuous $K_{1}$-g-frame sequence for $U$ with respect to $\left\{V_{\omega}\right\}_{\omega \in \Omega}$; then, $\left\{\Lambda_{\omega}\right\}_{\omega \in \Omega}$ is a C-tight continuous $K_{2}$-g-frame sequence for $U$ with respect to $\left\{V_{\omega}\right\}_{\omega \in \Omega}$ if and only if

$$
\mathscr{R}\left(\left(\frac{K_{2}^{*}}{\overline{\operatorname{span}}\left(\left\{\Lambda_{\omega}^{*}\left(V_{\omega}\right)\right\}_{\omega \in \Omega}\right)}\right)^{*}\right) \subset \mathscr{R}\left(\left(\frac{K_{1}^{*}}{\overline{\operatorname{span}}\left(\left\{\Lambda_{\omega}^{*}\left(V_{\omega}\right)\right\}_{\omega \in \Omega}\right)}\right)^{*}\right) .
$$

Proof. First, we suppose that $\Lambda=\left\{\Lambda_{\omega}\right\}_{\omega \in \Omega}$ is a $C$-tight continuous $K_{2}$-g-frame sequence for $U$ with respect to $\left\{V_{\omega}\right\}_{\omega \in \Omega}$; then, there exists $D>0$ such that

$D\left\|K_{2}^{*} x\right\|^{2} \leq \int_{\Omega}\left\|\Lambda_{\omega} x\right\|^{2} \mathrm{~d} \mu(\omega), \quad \forall x \in \overline{\operatorname{span}}\left(\left\{\Lambda_{\omega}^{*}\left(V_{\omega}\right)\right\}_{\omega \in \Omega}\right)$.
We have $\Lambda=\left\{\Lambda_{\omega}\right\}_{\omega \in \Omega}$ is a $C$-tight continuous $K_{1}$-gframe sequence for $U$ with respect to $\left\{V_{\omega}\right\}_{\omega \in \Omega}$; then, $\left\|K_{2}^{*} x\right\|^{2} \leq(C / D)\left\|K_{1}^{*} x\right\|^{2}$ for every $x \in \overline{\operatorname{span}}\left(\left\{\Lambda_{\omega}^{*}\left(V_{\omega}\right)\right\}_{\omega \in \Omega}\right)$.

\section{Hence,}

$$
\begin{array}{r}
\left\langle\left(\frac{K_{2}^{*}}{\overline{\operatorname{span}}\left(\left\{\Lambda_{\omega}^{*}\left(V_{\omega}\right)\right\}_{\omega \in \Omega}\right)}\right) x,\left(\frac{K_{2}^{*}}{\overline{\operatorname{span}}\left(\left\{\Lambda_{\omega}^{*}\left(V_{\omega}\right)\right\}_{\omega \in \Omega}\right)}\right) x\right\rangle=\left\langle K_{2}^{*} x, K_{2}^{*} x\right\rangle \\
\quad \leq \frac{C}{D}\left\langle K_{1}^{*} x, K_{1}^{*} x\right\rangle \\
=\frac{C}{D}\left\langle\left(\frac{K_{1}^{*}}{\overline{\operatorname{span}}\left(\left\{\Lambda_{\omega}^{*}\left(V_{\omega}\right)\right\}_{\omega \in \Omega}\right)}\right) x,\left(\frac{K_{1}^{*}}{\overline{\operatorname{span}}\left(\left\{\Lambda_{\omega}^{*}\left(V_{\omega}\right)\right\}_{\omega \in \Omega}\right)}\right) x\right\rangle .
\end{array}
$$

Therefore,

$$
\begin{aligned}
& \left(\frac{K_{2}^{*}}{\overline{\operatorname{span}}\left(\left\{\Lambda_{\omega}^{*}\left(V_{\omega}\right)\right\}_{\omega \in \Omega}\right)}\right)^{*}\left(\frac{K_{2}^{*}}{\overline{\operatorname{span}}\left(\left\{\Lambda_{\omega}^{*}\left(V_{\omega}\right)\right\}_{\omega \in \Omega}\right)}\right) \\
& \leq \frac{C}{D}\left(\frac{K_{1}^{*}}{\overline{\operatorname{span}}\left(\left\{\Lambda_{\omega}^{*}\left(V_{\omega}\right)\right\}_{\omega \in \Omega}\right)}\right)\left(\frac{K_{1}^{*}}{\overline{\operatorname{span}}\left(\left\{\Lambda_{\omega}^{*}\left(V_{\omega}\right)\right\}_{\omega \in \Omega}\right)}\right) .
\end{aligned}
$$

By Lemma 2, we have

$$
\mathscr{R}\left(\left(\frac{K_{2}^{*}}{\overline{\operatorname{span}}\left(\left\{\Lambda_{\omega}^{*}\left(V_{\omega}\right)\right\}_{\omega \in \Omega}\right)}\right)^{*}\right) \subset \mathscr{R}\left(\left(\frac{K_{1}^{*}}{\overline{\operatorname{span}}\left(\left\{\Lambda_{\omega}^{*}\left(V_{\omega}\right)\right\}_{\omega \in \Omega}\right)}\right)^{*}\right) .
$$

Conversely,

suppose $\left.\left.\left(\left\{\Lambda_{\omega}^{*}\left(V_{\omega}\right)\right\}_{\omega \in \Omega}\right)\right)^{*}\right) \subset \mathscr{R}\left(\left(K_{1}^{*} / \overline{\operatorname{span}}\left(\left\{\Lambda_{\omega}^{*}\left(V_{\omega}\right)\right\}_{\omega \in \Omega}\right)^{*}\right)\right.$.

By Lemma 2, there exists $\lambda>0$ such that

$$
\begin{aligned}
& \left(\frac{K_{2}^{*}}{\overline{\operatorname{span}}\left(\left\{\Lambda_{\omega}^{*}\left(V_{\omega}\right)\right\}_{\omega \in \Omega}\right)}\right)^{*}\left(\frac{K_{2}^{*}}{\overline{\operatorname{span}}\left(\left\{\Lambda_{\omega}^{*}\left(V_{\omega}\right)\right\}_{\omega \in \Omega}\right)}\right) \\
& \leq \lambda\left(\frac{K_{1}^{*}}{\overline{\operatorname{span}}\left(\left\{\Lambda_{\omega}^{*}\left(V_{\omega}\right)\right\}_{\omega \in \Omega}\right)}\right)\left(\frac{K_{1}^{*}}{\overline{\operatorname{span}}\left(\left\{\Lambda_{\omega}^{*}\left(V_{\omega}\right)\right\}_{\omega \in \Omega}\right)}\right),
\end{aligned}
$$

so for every $x \in \overline{\operatorname{span}}\left(\left\{\Lambda_{\omega}^{*}\left(V_{\omega}\right)\right\}_{\omega \in \Omega}\right)$, we have 


$$
\begin{aligned}
\left\|K_{2}^{*} x\right\|^{2} & =\left\langle\left(\frac{K_{2}^{*}}{\overline{\operatorname{span}}\left(\left\{\Lambda_{\omega}^{*}\left(V_{\omega}\right)\right\}_{\omega \in \Omega}\right)}\right)^{*}\left(\frac{K_{2}^{*}}{\overline{\operatorname{span}}\left(\left\{\Lambda_{\omega}^{*}\left(V_{\omega}\right)\right\}_{\omega \in \Omega}\right)}\right) x, x\right\rangle \\
& \leq \lambda\left(\frac{K_{1}^{*}}{\overline{\operatorname{span}}\left(\left\{\Lambda_{\omega}^{*}\left(V_{\omega}\right)\right\}_{\omega \in \Omega}\right)}\right)\left(\frac{K_{1}^{*}}{\overline{\operatorname{span}}\left(\left\{\Lambda_{\omega}^{*}\left(V_{\omega}\right)\right\}_{\omega \in \Omega}\right)}\right) \\
& =\lambda\left\|K_{1}^{*} x\right\|^{2}=\frac{\lambda}{c} \int_{\Omega}\left\|\Lambda_{\omega} x\right\|^{2} \mathrm{~d} \mu(\omega) .
\end{aligned}
$$

Then, for all $x \in \overline{\operatorname{span}}\left(\left\{\Lambda_{\omega}^{*}\left(V_{\omega}\right)\right\}_{\omega \in \Omega}\right)$,

$$
\frac{c}{\lambda}\left\|K_{2}^{*} x\right\|^{2} \leq \int_{\Omega}\left\|\Lambda_{\omega} x\right\|^{2} \mathrm{~d} \mu(\omega) \leq C\left\|K_{1}^{*}\right\|^{2}\|x\|^{2} .
$$

This completes the proof.

\section{Conclusion}

In this article, we have proved some properties of dual continuous $\mathrm{K}$-g-frames in Hilbert spaces. These results are extensions of the related results announced in [8]. The presented theorems extend, generalize, and improve many existing results in the literature.

\section{Data Availability}

No data were used to support this study.

\section{Conflicts of Interest}

The authors declare no conflicts of interest.

\section{References}

[1] R. J. Duffin and A. C. Schaeffer, "A class of nonharmonic fourier series," Transactions of the American Mathematical Society, vol. 72, no. 2, p. 341, 1952.

[2] I. Daubechies, A. Grossmann, and Y. Meyer, "Painless nonorthogonal expansions," Journal of Mathematical Physics, vol. 27, no. 5, pp. 1271-1283, 1986.

[3] D. Gabor, "Theory of communication. Part 1: the analysis of information," Journal of the Institution of Electrical Engineers Part III: Radio and Communication Engineering, vol. 93, no. 26, pp. 429-441, 1946.

[4] M. R. Abdollahpour and M. H. Faroughi, "Continuous G-frames in Hilbert space," Southeast Asian Bulletin of Mathematics, vol. 32, pp. 1-19, 2008.

[5] E. Alizadeh, A. Rahimi, E. Osgooei, and M. Rahmani, "Continuous K-g-Frames in Hilbert spaces," Bulletin of the Iranian Mathematical Society, vol. 45, no. 4, p. 1091, 2018.

[6] O. Christensen, An Introduction to Frames and Riesz Bases, Birkhäuser, Boston, MA, USA, 2003.

[7] R. G. Douglas, "On majorization, factorization, and range inclusion of operators on Hilbert space," Proceedings of the American Mathematical Society, vol. 17, no. 2, p. 413, 1966.

[8] Z. Q. Xiang, "Canonical dual K-g-Bessel sequences and K-gFrame sequences," Results Math, vol. 73, p. 17, 2018.

[9] R. Paley and N. Wiener, Fourier Transforms in Complex Domains, MAS Colloquium Publications, 1934. 